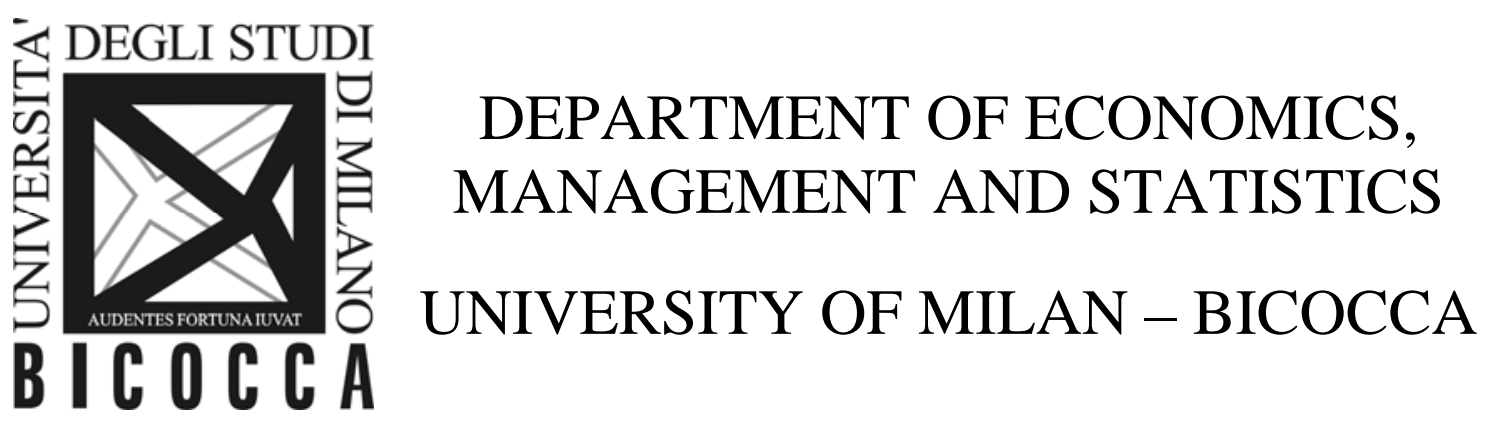

DEMS WORKING PAPER SERIES

\title{
A cobweb model with alternating demand and supply functions
}

\author{
Fausto Cavalli
}

No. 325 - February 2016

Dipartimento di Economia, Metodi Quantitativi e Strategie di Impresa Università degli Studi di Milano - Bicocca

http://dems.unimib.it/ 


\title{
A cobweb model with alternating demand and supply functions
}

\author{
Fausto Cavalli*
}

\begin{abstract}
In this work I present a cobweb model for markets characterized by two couples of demand and supply functions which cyclically alternate with period two, in a succession of peak and off-peak market phases. Starting from classical adaptive expectations, a new expectation formation mechanism is presented, to take into account such markets' peculiarity. In particular, to adapt the previous in-phase expected price, agents use both in-phase and out-of-phase expectation errors, suitably weighted through a phase weight. It is shown that the resulting model is described by a nonautonomous difference equation. The local asymptotic stability of the steady state equilibrium is studied, showing that it depends on the expectation weight, the phase weight and on both the relative slopes, at the equilibrium, of the supply functions with respect to the demand functions. Several crucial differences with respect to the classical cobweb model are highlighted, showing the potentially ambiguous role of expectation weight and of relative slopes. It is shown that destabilization can occur both through a flip and a Neimark-Sacker bifurcation, which can occur for the same market conditions and different expectation weights.
\end{abstract}

Keywords: Cobweb model, cyclic demand and supply, non-autonomous equations, complex dynamics

JEL classification: E32, D84, C60, C62

\section{Introduction}

The cobweb model was introduced to provide a theoretical explanation of temporary equilibrium fluctuations in a single market with one lag in supply. Seminal contributions about this modelling approach can be found in the works by Kaldor [1] and Ezekiel [2], in which the so-called "Cobweb Theorem" is presented. It is shown how, in a purely competitive market in which there is a lag between production decisions and their realization and where the available supply sets the price, fluctuating price dynamics may occur, which can either

\footnotetext{
*Address: Department of Economics, Management and Statistics, University of Milano-
} Bicocca, Piazza dell'Ateneo Nuovo 1, 20126, Milano, Italy, e-mail: fausto.cavalli@unimib.it 
converge, exhibit a persistent period-two cycle or diverge. Essential roles for the resulting dynamics are played by both market's and economic agents' characteristics. The former are summarized by the shape of demand and supply curves in their intersection point, the (steady state) equilibrium. The latter are encompassed by the way in which suppliers decide, at a generic period $t$, the quantity to produce, which will be supplied in the next period $t+1$. This is the expectation formation mechanism, that, depending on the degree of rationality assumed for the suppliers, describes how next period expected prices are formed. In [2] naive expectations are assumed, namely agents expect that next period price will be the same as in the current one. With this assumption, the cobweb theorem says that if the elasticity of supply at the equilibrium point is smaller, in absolute value, than the elasticity of demand, convergent price dynamics occur, while, when the converse is true, prices diverge. In the limit situation in which the two elasticities are identical, a period-two price cycle arises.

Since the literature about cobweb models is tremendously wide, I shall just mention some milestone contributions of the research strands the present work belongs to.

A first research strand concerns the study of boundedly rational expectation mechanisms and their role on the resulting dynamics. Nerlove [3] considered a cobweb model with linear demand and supply functions in which agents adapt the production level on the basis of the so called expectation error, namely, on the difference between the last expected and realized prices, regulated by an expectation weight. The resulting expectation formation mechanism is called "adaptive expectations", and it provides a simple example of a more refined, with respect to naive expectations, expectation mechanism, based on a boundedly rational learning rule. Cobweb models under adaptive expectations and for different demand and supply functions were then investigated to study the possible resulting dynamical behaviors, as for example in $[4,5,6]$. Briefly, the results can be summarized as follows. Stability of the steady state is regulated by the size of the expectation weight and by the relative slope of the supply function with respect to that of the demand function. For any relative slope, there always exists an interval of sufficiently small expectation weights for which price dynamics are convergent. Conversely, if too relevance is given to expectation errors, especially when the relative slope is sufficiently large, periodic and chaotic dynamics can arise.

A second research strand aims to extend to more general economic settings the cobweb model approach. I just mention the contribution by Dieci and Westerhoff [7], in which the classical cobweb is enriched by allowing producers to enter different markets, so that the interaction between markets is taken into account in the model. In [7] it is shown that this allows the occurrence of quasi-periodic motions in the resulting dynamics. The previous model was then applied to a particular market example by Lundberg et al. in [8].

The present contribution belongs to both the above research strands. The main goal of this paper is to introduce and study a new theoretical framework to analyze a class of markets characterized by demand/supply cyclical oscillations over time. The real world prototypical example is represented by electricity 
markets, a first cobweb model of which was proposed by Cavalli et al. in [9]. In such markets the exchanged good is unique but demand and supply curves substantially vary over time, following a well known profile characterized by a deterministic cyclical recurrence. In particular, in this paper I will focus on the period-2 case, in which two couples of different demand and supply functions cyclically alternate, in order to resemble the alternation of peak and off-peak phases in a cyclical market. The considered modelling framework also requires to introduce a new expectation mechanism, consistent the market assumptions. Since it is realistic to suppose that agents are aware of the cyclical nature of the market, a new expectation formation mechanism is taken into account, starting from classical adaptive expectations, that, in their original formulation, are not consistent with the considered market and require to be modified to fit with the cyclical demand/supply assumption. It is then assumed that agents, in order to form their expectations about next period prices, adapt their in-phase expected price on the basis of both in-phase and out-of-phase expectation errors, which are suitably weighted. This means that if, for instance, at time $\tau+1$ market is in a peak phase, agents, in order to form the expected price $\pi_{\tau+1}$, do not adapt the expected price at time $\tau$, since at $\tau$ the market is in the off-peak phase, but conversely, adapt the expected price at time $\tau-1$, in which market is inphase with time $\tau+1$. Moreover, it is assumed that, besides the usual level of confidence given to expectation errors, which is summarized by the expectation weight $\omega$, agents attribute different significance to in-phase errors with respect to out-of-phase errors, through a phase weight $\nu$.

The resulting model is very different from the classical cobweb model. Firstly, it results in a non-autonomous difference equation. It's worth mentioning that other non-autonomous cobweb models can be found in the literature. For example, it is well known that if memory is taken into account in a learning process, as in $[10,11,12]$, then the resulting difference equation is non-autonomous. Some examples of cobweb models with memory in the learning process are $[13,14,15,16]$. However, in these works the non-autonomous nature of the resulting equation is due to a modification of the expectation formation mechanism. In the present contribution it is an intrinsic result of the alternation of demand and supply, and hence it is ascribable to the market structure, which then affects the expectation formation mechanism, too.

The main theoretical novelties concern the effect of $\omega, \nu$ and relative slopes $a_{i}$ of supply and demand functions on the local asymptotic stability of the steady state equilibrium. Despite some points of contact, the role of the expectation weight exhibits substantial differences with respect to that in the classical cobweb model, which can be summarized as follows: 


\begin{tabular}{|c|c|}
\hline Present cobweb model & Classical cobweb model \\
\hline $\begin{array}{l}\text { Both a flip and a Neimark-Sacker bi- } \\
\text { furcations can occur }\end{array}$ & Only a flip bifurcation can $\mathrm{o}$ \\
\hline $\begin{array}{l}\text { Up to three stability thresholds are } \\
\text { possible }\end{array}$ & $\begin{array}{l}\text { At most one } \mathrm{s} \\
\text { possible }\end{array}$ \\
\hline $\begin{array}{l}\text { Increasing the expectation weight, a } \\
\text { stable steady state equilibrium can } \\
\text { lose stability and an unstable one } \\
\text { can recover stability }\end{array}$ & $\begin{array}{l}\text { Increasing the expectation weight, a } \\
\text { stable steady state equilibrium can } \\
\text { lose stability while an unstable one } \\
\text { remains unstable }\end{array}$ \\
\hline $\begin{array}{l}\text { Decreasing the expectation weight, } \\
\text { a stable steady state equilibrium } \\
\text { can become unstable and an unsta- } \\
\text { ble one can recover stability }\end{array}$ & $\begin{array}{l}\text { Decreasing the expectation weight, } \\
\text { a stable steady state equilibrium re- } \\
\text { mains stable and an unstable one } \\
\text { can recover stability }\end{array}$ \\
\hline & \\
\hline
\end{tabular}
dynamics can simultaneously occur for different expectations weights

Similarly, the role of relative slopes at the equilibrium is ambiguous too. Differently from the classical cobweb model, in which steady state can lose stability when relative slopes increase, in a double phase cobweb model we can have situations in which increasing one of the two relative slopes allows the steady state equilibrium to recover stability. Finally, the role of phase weight is less ambiguous, as it is generally destabilizing. Since decreasing $\nu$ actually corresponds to increase the coupling degree between the two phases of the market, the previous stability result says that stability improves as coupling between phases becomes increasingly stronger.

The remainder of the paper is organized as follows. In Section 2, the model is introduced. In Section 3, stability results for the steady state equilibrium are presented and compared to those of the classical cobweb model. In Section 4 some research perspectives are outlined. Proofs are collected in Appendix.

\section{Model}

In this section I introduce a theoretical cobweb model for a single good market characterized by two couples of demand and supply functions which cyclically alternate. Throughout the paper, this kind of market will be called double phase market.

The whole market demand function can be represented by introducing the time periodic function $D: I \times \mathbb{N}^{+} \rightarrow \mathbb{R}^{+}$defined by

$$
D\left(p_{\tau}, \tau\right)= \begin{cases}D_{1}\left(p_{\tau}\right) & \text { if } \tau \text { is odd } \\ D_{2}\left(p_{\tau}\right) & \text { if } \tau \text { is even }\end{cases}
$$

where $p_{\tau}$ is the market price at $\tau$. Likewise, we can introduce the time periodic 
supply function $S: J \times \mathbb{N}^{+} \rightarrow \mathbb{R}^{+}$, defined by

$$
S\left(p_{\tau}, \tau\right)= \begin{cases}S_{1}\left(p_{\tau}\right) & \text { if } \tau \text { is odd } \\ S_{2}\left(p_{\tau}\right) & \text { if } \tau \text { is even }\end{cases}
$$

We assume that odd/even times demand functions $D_{i}: I \rightarrow \mathbb{R}^{+}, i=1,2$, where $I$ is a suitable interval, are smooth and decreasing functions. Similarly, we assume that odd/even times supply functions $S_{i}: J \rightarrow \mathbb{R}^{+}, i=1$, 2 , where $J$ is a suitable interval, are smooth increasing functions. For the sake of simplicity, in this section we assume that all the demand (resp. supply) functions share the same domain, but such assumption is not essential and can be easily removed.

At any generic time $\tau$, the market is characterized by the same couple of demand/supply functions which occurs at times $\tau+2 n, n \in \mathbb{Z}$. This allows saying that two times $\tau_{2}>\tau_{1}$ are in-phase when they both are either odd or even. Conversely, we say that $\tau_{1}$ and $\tau_{2}$ are out-of-phase.

I stress that at any set of 2 consecutive times $\tau, \tau+1$ the market is characterized by (possibly) different demand $D_{i_{1}}, D_{i_{2}}$ and/or supply $S_{i_{1}}, S_{i_{2}}$ functions, where index vector $\left(i_{1}, i_{2}\right)$ can be either $(1,2)$ or $(2,1)$. Finally, without loss of generality, in (1) we assume that for $\tau=1$ the market is characterized by demand function $D_{1}$ and supply function $S_{1}$. The demanded quantity at each $\tau$ is then

$$
q_{\tau}^{D}=D\left(p_{\tau}, \tau\right),
$$

while the supplied quantity $q_{\tau}^{S}$ at $\tau$ is

$$
q_{\tau}^{S}=S\left(\pi_{\tau}, \tau\right),
$$

where $\pi_{t}$ is the price that agents expect for time $\tau$.

I remark that the shapes of both demand and supply functions, as well as their domains $I$ and $J$, depend on the institutional characteristics of the particular market under consideration. In this paper I only deal with generic functions, assuming that each function $D_{i}$ always has one intersection with the corresponding function $S_{i}$.

To complete the model I need to specify the expectation formation mechanism. To this end, in agreement with the classical cobweb framework, I assume that suppliers, in order to decide the quantity for time $\tau+1$, form their price expectations on the basis of the information collected from the most recent times. In the literature, the research about expectation formation mechanisms is very wide and several increasingly refined expectation formation mechanisms have been studied. In particular, adaptive expectations $[3,4,6,17]$ are a significant example of non-rational behavior, in which agents form their expectations about the future price starting from the current expected price and correcting it on the basis of the expectation error (i.e. the difference between the realized and the expected prices), regulated by an expectation weight $\omega$.

More generally, for a double phase market, it is reasonable to assume that agents are aware of the cyclical nature of demand and supply functions, so that they know that time $\tau+1$ the market will be in phase with time $\tau-1$ (and not 
with $\tau$ ). I then suppose that expected price $\pi_{\tau+1}$ is formed adapting the previous in-phase expected price $\pi_{\tau-1}$ on the basis of the past expectation errors. In particular, I assume that both in-phase, $p_{\tau-1}-\pi_{\tau-1}$, and out-of-phase, $p_{\tau}-\pi_{\tau}$, expectation errors are used. The resulting double phase adaptive expectation formation mechanism is then given by

$$
\pi_{\tau+1}=\pi_{\tau-1}+\nu \omega\left(p_{\tau-1}-\pi_{\tau-1}\right)+(1-\nu) \omega\left(p_{\tau}-\pi_{\tau}\right)
$$

where $\omega \in(0,1]$ is the expectation weight and $0 \leq \nu \leq 1$ is the phase-weight, which specifies the relevance given to the in-phase error. I notice that expected price $\pi_{\tau-1}$ is adapted through a weighted average of phase expectation errors.

Since in-phase error $p_{\tau-1}-\pi_{\tau-1}$ is the most significant for the determination of $\pi_{\tau+1}$, I assume that $\nu>1 / 2$. Phase-weight $\nu$ is also (inversely) connected to the degree of coupling of the different market phases, which is null when $\nu=1$ (since no relevance is given to out-of-phase errors) and maximum when $\nu \approx 1 / 2$ (since in-phase and out-of-phase errors approximatively have the same relevance). Moreover, for $\omega=0$, as for classical adaptive expectations, we would actually have no dynamic adjustment, since $\pi_{\tau+1}=\pi_{\tau-1}$. Finally, I notice that the considered theoretical framework includes the very particular situation in which $D_{1} \equiv D_{2}$ and $S_{1} \equiv S_{2}$, where we actually do not have a double phase market and both demand and supply functions, as in the classical cobweb model, do not directly depend on $\tau$.

Imposing, at each time $\tau$, temporary equilibrium condition $D\left(p_{\tau}, \tau\right)=S\left(\pi_{\tau}, \tau\right)$, we obtain

$$
p_{\tau}=D^{-1}\left(S\left(\pi_{\tau}, \tau\right), \tau\right)
$$

where $D^{-1}\left(q_{\tau}, \tau\right)$ is the inverse function of $D$ with respect to its first component, namely

$$
D^{-1}\left(q_{\tau}, \tau\right)= \begin{cases}D_{1}^{-1}\left(q_{\tau}\right) & \text { if } \tau \text { is odd } \\ D_{2}^{-1}\left(q_{\tau}\right) & \text { if } \tau \text { is even. }\end{cases}
$$

Combining (3) and (4) we obtain the double phase cobweb model, represented by the second order non-autonomous difference equation

$$
\begin{aligned}
\pi_{\tau+1}= & \pi_{\tau-1}+\nu \omega\left(D^{-1}\left(S\left(\pi_{\tau-1}, \tau-1\right), \tau-1\right)-\pi_{\tau-1}\right) \\
& +(1-\nu) \omega\left(D^{-1}\left(S\left(\pi_{\tau}, \tau\right), \tau\right)-\pi_{\tau}\right)
\end{aligned}
$$

given some $\pi_{0}$ and $\pi_{-1}$. Other examples of cobweb models based on nonautonomous equations can be found in $[13,18,14,10,15,16]$. I notice that, contrary to the previous literature, the non-autonomous nature of the present model is intrinsically connected with the cyclicity of demand/supply functions characterizing the market itself. Such cyclicity indeed induces the peculiar form of adaptive expectations, which, in turn, strengthen the presence of nonautonomous terms in (5). Conversely, in the above mentioned works, the nonautonomous model is induced by the generalization, which includes memory effects, of the expectation formation mechanisms. 
In general, non-autonomous equation (5) does not possess a steady state, because of the cyclicity of the demand and supply functions, while it always exhibits a steady cycle of period 2, characterized by the alternation of the two quantities that realize

$$
D\left(p^{i, *}, i\right)=S\left(p^{i, *}, i\right), i=1,2 .
$$

To study stability, it is expedient to rewrite equation (5) as an autonomous two-dimensional system, in which each component of the vector steady state corresponds to an element of the period-2 steady cycle of (5). To avoid ambiguous notation, $t$ is used for the sequence of times of the autonomous system, in which we consider the evolution of two dimensional vectors $\vec{\pi}_{t}$ consisting of two expected prices, so that the $i$ th element of $\vec{\pi}_{t}$ is the expected price of the $i$ th phase of the market. Indeed, from $\vec{\pi}_{t}$ we can obtain the corresponding vectors of realized prices and of traded quantities.

To write the autonomous system, I introduce function $\Phi: I^{2} \times \mathbb{N} \rightarrow\left(\mathbb{R}^{+}\right)^{2}$ defined by $\left(\rho_{\tau+1}^{1}, \rho_{\tau+1}^{2}\right)=\Phi\left(\rho_{\tau}^{1}, \rho_{\tau}^{2}, \tau\right)$ through

$$
\begin{aligned}
& \rho_{\tau+1}^{1}=\rho_{\tau}^{2}+(1-\nu) \omega\left(D^{-1}\left(S\left(\rho_{\tau}^{1}, \tau\right), \tau\right)-\rho_{\tau}^{1}\right), \\
& \rho_{\tau+1}^{2}=\rho_{\tau}^{1}+\nu \omega\left(D^{-1}\left(S\left(\rho_{\tau}^{1}, \tau\right), \tau\right)-\rho_{\tau}^{1}\right) .
\end{aligned}
$$

and functions $\Phi_{i}: I^{2} \rightarrow\left(\mathbb{R}^{+}\right)^{2}$ defined by $\Phi_{i}\left(\vec{\rho}_{\tau}\right)=\Phi\left(\vec{\rho}_{\tau}, i\right)$. I stress that $\rho^{i}$ are auxiliary variables, which are temporarily used to introduce the autonomous system. Finally, I introduce $T: I^{2} \rightarrow\left(\mathbb{R}^{+}\right)^{2}$ defined by

$$
T(\vec{\pi})=\Phi_{2} \circ \Phi_{1}(\vec{\pi}),
$$

which gives

$$
\begin{aligned}
\pi_{t+1}^{1}= & \pi_{t}^{1}+\nu \omega\left(D_{1}^{-1}\left(S_{1}\left(\pi_{t}^{1}\right)\right)-\pi_{t}^{1}\right) \\
& +(1-\nu) \omega\left(D_{2}^{-1}\left(S_{2}\left(\pi_{t}^{2}+(1-\nu) \omega\left(D_{1}^{-1}\left(S_{1}\left(\pi_{t}^{1}\right)\right)-\pi_{t}^{1}\right)\right)\right)\right. \\
& \left.-\left(\pi_{t}^{2}+(1-\nu) \omega\left(D_{1}^{-1}\left(S_{1}\left(\pi_{t}^{1}\right)\right)-\pi_{t}^{1}\right)\right)\right) \\
\pi_{t+1}^{2}= & \pi_{t}^{2}+(1-\nu) \omega\left(D_{1}^{-1}\left(S_{1}\left(\pi_{t}^{1}\right)\right)-\pi_{t}^{1}\right) \\
& +\nu \omega\left(D_{2}^{-1}\left(S_{2}\left(\pi_{t}^{2}+(1-\nu) \omega\left(D_{1}^{-1}\left(S\left(\pi_{t}^{1}\right)\right)-\pi_{t}^{1}\right)\right)\right)\right. \\
& \left.-\left(\pi_{t}^{2}+(1-\nu) \omega\left(D_{1}^{-1}\left(S_{1}\left(\pi_{t}^{1}\right)\right)-\pi_{t}^{1}\right)\right)\right)
\end{aligned}
$$

It is easy to see that $\tau$ and $t$ are indeed linked and we can unambiguously move from $\tau$ to $t$ and vice versa. The $i$-th phase of time $t$ corresponds to time 
$\tau=2(t-1)+i$. Conversely, from time $\tau$, we can unequivocally obtain the corresponding time $t$ and phase $i$ from

$$
t=\lfloor\tau / 2\rfloor+1, \quad i=\tau-2(t-1),
$$

where $\lfloor z\rfloor$ stands for the largest integer not exceeding $z$.

I notice that System (8) consists of two coupled equations. Since in the extreme case of $\nu=1$, expectation mechanism (3) simplifies to

$$
\pi_{\tau+1}=\pi_{\tau-1}+\omega\left(p_{\tau-1}-\pi_{\tau-1}\right),
$$

model (8) reduces to the diagonal system

$$
\pi_{t+1}^{i}=\pi_{t}^{i}+\omega\left(D_{i}^{-1}\left(S_{i}\left(\pi_{t}^{i}\right)\right)-\pi_{t}^{i}\right), \quad i=1,2 .
$$

In this case we actually have two distinct, independent equations (which provide independent dynamics), as (10) is the classical adaptive expectation formation mechanism and $\pi_{\tau+1}^{i}$ in (11) only depend on the in-phase previous expected price. Actually, when $\nu=1$ the model can be assimilated to two independent classical cobweb models with adaptive expectations. In this case we can also have that just one $\pi^{i, *}$ is stable. For these reasons, I consider the case $\nu=1$ as modeled by two independent equations (and not by a single diagonal system), while I consider all the remaining cases with $\nu \neq 1$ as represented by a single two-dimensional system. I will respectively refer to (7) for $\nu<1$ and to (11) as to coupled and uncoupled models, and by saying coupled (respectively uncoupled) phases I will refer to each phase of the coupled (respectively uncoupled) models. I will be mainly interested in studying the behavior of the coupled model, while the uncoupled model will be considered as an intermediate situation between the classical single phase and the uncoupled double phase cobweb models.

I notice that both in this and in the following section we shall deal with a deterministic cobweb model. A stochastic perturbation term may be easily added, as well.

\section{$3 \quad$ Stability analysis}

In this section I analytically investigate the double phase cobweb model introduced in the previous section, trying to disentangle the role of each parameter and to clarify the reasons for the occurrence of each new behavior, in order to propose an interpretation of the results with respect to the classical cobweb model.

I recall that, as already noticed, the degree of coupling between phases inversely depends on phase-weight $\nu$, and that one can speak of uncoupled phases when the phase-weight is maximum $(\nu=1)$, of weak coupling of phases for $\nu \approx 1$ and of strong coupling for $\nu \rightarrow 1 / 2$ when each phase error is approximatively given the same relevance. 
Since I aim at understanding the effects introduced in the dynamics by the cyclicity of demand and supply functions, it is worth recalling the stability results for the classical cobweb model with either static or adaptive expectations ${ }^{1}$. In particular, for the classical cobweb model with static expectations, a steady state equilibrium price $p^{*}$ is locally asymptotically stable if

$$
-1<\frac{S^{\prime}\left(p^{*}\right)}{D^{\prime}\left(p^{*}\right)}(<1),
$$

namely if, at $p^{*}$, the slope of the supply function is sufficiently small with respect to the absolute value of the slope of the demand function. In the case of adaptive expectations, the previous result becomes

$$
1-\frac{2}{\omega}<\frac{S^{\prime}\left(p^{*}\right)}{D^{\prime}\left(p^{*}\right)}(<1) .
$$

The rightmost inequalities in both (12) and (13) are always fulfilled since $S^{\prime} / D^{\prime}$ is negative. The role of $\omega$ on steady state stability in the classical cobweb model can then be summarized as follows. Concerning the local stability of the steady state, I recall $([2,3,4])$ that

- steady state stability depends on the relative slope of the supply function with respect to that of the demand function;

- there always exists a sufficiently small positive $\hat{\omega}$ so that the steady state is stable for $\omega<\hat{\omega}$;

- if the steady state is stable for some $\hat{\omega}$, then it is automatically stable for any $\omega<\hat{\omega}$;

- if adaptive expectations provide unstable dynamics for some $\omega \in(0,1]$, then static expectations provide unstable dynamics, too.

When stability is lost, we have ([6]) that

- with static expectations, either a period-2 cycle or diverging trajectories occur;

- adaptive expectations can give rise to periodic and chaotic dynamics for suitable classes of supply functions.

Before investigating local stability, I show that $\vec{p}^{*}$ is the unique steady state of (8), which, thanks to the considerations of the previous section, guarantees that (3) has the only period-2 cycle $\left(p^{1, *}, p^{2, *}\right)$.

Proposition 1. The only steady state of system (8) is $\vec{p}^{*}=\left(p^{1, *}, p^{2, *}\right)$.

\footnotetext{
${ }^{1}$ For seminal results about the possible dynamic behaviors of classical linear and nonlinear cobweb models, we refer to $[2,3,4,6,17]$.
} 
The previous proposition guarantees that expectation formation mechanism (3) preserves the steady state equilibrium. We notice that, in the proof, I used $\nu \neq 1 / 2$. If we allowed $\nu=1 / 2$ (arithmetic mean of errors), System (8) would have additional, spurious, steady states.

In order to write stability conditions for $\vec{p}^{*}$ in a more compact form, I introduce the relative slopes of functions $S_{i}$ with respect to $D_{i}$ at $p^{i, *}$

$$
a_{1}=\frac{S_{1}^{\prime}\left(p^{1, *}\right)}{D_{1}^{\prime}\left(p^{1, *}\right)}, \quad a_{2}=\frac{S_{2}^{\prime}\left(p^{2, *}\right)}{D_{2}^{\prime}\left(p^{2, *}\right)} .
$$

It is easy to see that $\left|a_{i}\right|, i=1,2$ correspond to the ratio between the elasticity of supply and of demand. In brief, in what follows we will refer to each $a_{i}$ as to relative slope or relative elasticity. I notice that, from the assumptions on $S_{i}$ and $D_{i}$, we have $a_{i}<0$. The local asymptotic stability of $\vec{p}^{*}$ is studied in the following proposition.

Proposition 2. Steady state equilibrium $\vec{p}^{*}$ is locally asymptotically stable provided that

$$
\begin{aligned}
& \left(1-a_{1}\right)\left(1-a_{2}\right) \omega^{2}(2 \nu-1)>0, \\
& \nu \omega\left(2-a_{1}-a_{2}-\nu \omega\left(1-a_{1}\right)\left(1-a_{2}\right)\right)>0, \\
& \left(1-a_{1}\right)\left(1-a_{2}\right)\left(2 \nu^{2}-2 \nu+1\right) \omega^{2}-2 \nu\left(2-a_{1}-a_{2}\right) \omega+4>0 .
\end{aligned}
$$

The previous result is actually the generalization of the stability condition (13) to double phase markets. From conditions (15) we have that, in agreement with the classical cobweb model, local stability depends on the expectation weight $\omega$ and on the reactivity of the market at the steady state. However, the periodicity of demand and supply functions makes necessary to deal with two, possibly different, slopes at each $\pi^{i *}$. Finally, in the present model, stability is also affected by phase-weight $\nu$. The existence of two phases allows agents to decide which weights to assign to the errors of each single phase. Phase error weights in turn introduce a coupling between phases.

Since $a_{i}<0$, we have that (15a) is satisfied provided that $\nu>1 / 2$. This means that the assumptions on phase-weights actually coincide with the essential requirement on $\nu$ to have stability. I recall that, from bifurcation theory, when condition (15b) (respectively $(15 c)$ ) is violated, the steady state loses its stability through a Neimark-Sacker (resp. period-doubling) bifurcation (see [19]). Due to the linear (15b) and quadratic (15c) conditions in both $\omega$ and $\nu$, I can have up to three stability thresholds. However, the actual occurrence of different kinds of instability and the presence of multiple stability thresholds depends on the parameter choice and will be clarified in Sections 3.1 and 3.2.

In the remainder of this section I will investigate the role of $\omega, \nu$ and of $a_{1}, a_{2}$ on the steady state stability. I remark that $\omega$ and $\nu$ are connected to the agents' behavior, as the expectation formation mechanism depends on them, while $a_{1}$ and $a_{2}$ take into account all the market characteristics and encompass the effect of parameters on which demand and supply depend. To describe the possible dynamical behaviors with respect to a parameter $\xi$ (which will be either $\omega, \nu$ or 


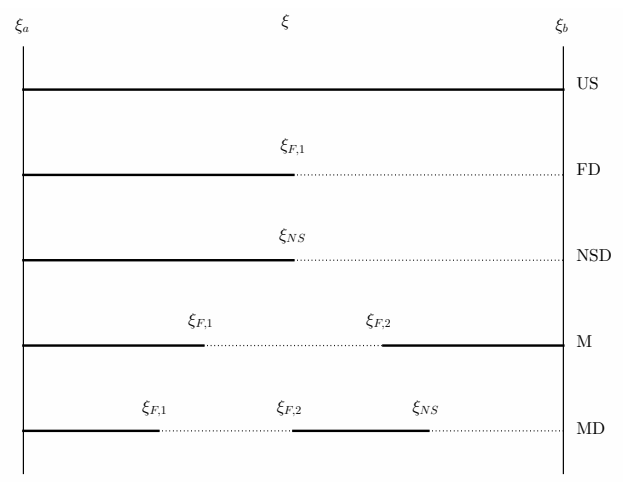

Figure 1: Possible stability scenarios with respect to a parameter $\xi$. A solid (respectively dotted) line is used for stability (respectively instability) intervals.

$\left.a_{i}\right)$, I introduce several scenarios. To this end, I assume that $\xi$ varies within an interval $I$ with endpoints $\xi_{a}$ and $\xi_{b}$. I describe scenarios for the case of an open interval $\left(\xi_{a}, \xi_{b}\right)$; the same definitions can be easily adapted to include endpoints, too. I say that we have

- a flip destabilizing scenario (FD), if there exists $\xi_{F} \in\left(\xi_{a}, \xi_{b}\right)$ such that $\vec{p}^{*}$ is stable for $\xi \in\left(\xi_{a}, \xi_{F}\right)$ and unstable for $\xi \in\left(\xi_{F}, \xi_{b}\right)$, and for $\xi=\xi_{F}$ stability is lost through a flip bifurcation;

- a Neimark-Sacker destabilizing scenario (NSD), if there exists $\xi_{N S} \in$ $\left(\xi_{a}, \xi_{b}\right)$ such that $\vec{p}^{*}$ is stable for $\xi \in\left(\xi_{a}, \xi_{N S}\right)$ and unstable for $\xi \in$ $\left(\xi_{N S}, \xi_{b}\right)$, and for $\xi=\xi_{N S}$ stability is lost through a Neimark-Sacker bifurcation;

- a mixed scenario (M), if there exist $\xi_{F, 1}<\xi_{F, 2}$, with $\xi_{F, i} \in\left(\xi_{a}, \xi_{b}\right)$, such that $\vec{p}^{*}$ is stable for $\xi \in\left(\xi_{a}, \xi_{F, 1}\right) \cup\left(\xi_{F, 2}, \xi_{b}\right)$ and unstable for $\xi \in\left(\xi_{F, 1}, \xi_{F, 2}\right)$ and for $\xi=\xi_{F, 1}$ (resp. $\xi=\xi_{F, 2}$ ) stability is lost (resp. recovered) through a period-doubling (resp. halving) bifurcation;

- a mixed-destabilizing scenario (MD), if there exist $\xi_{F, 1}<\xi_{F, 2}<\xi_{N S}$, with $\xi_{F, i} \in\left(\xi_{a}, \xi_{b}\right), \xi_{N S} \in\left(\xi_{a}, \xi_{b}\right)$ such that $\vec{p}^{*}$ is stable for $\xi \in\left(\xi_{a}, \xi_{F, 1}\right) \cup$ $\left(\xi_{F, 2}, \xi_{N S}\right)$ and unstable for $\xi \in\left(\xi_{F, 1}, \xi_{F, 2}\right) \cup\left(\xi_{N S}, \xi_{b}\right)$ and for $\xi=\xi_{F, 1}$ (resp. $\left.\xi=\xi_{F, 2}\right)$ stability is lost (resp. recovered) through a perioddoubling (resp. halving) bifurcation, while for $\xi=\xi_{N S}$ stability is lost trough a Neimark-Sacker bifurcation;

- an unconditionally stable scenario (US), if $\vec{p}^{*}$ is stable for any $\xi \in\left(\xi_{a}, \xi_{b}\right)$.

The previous scenarios are graphically illustrated in Figure 1. In the following subsections I study stability with respect to each parameter $\omega, \nu$ and $a_{i}$. The aim is to show the occurrence of the possible stability scenarios. 


\subsection{Stability with respect to expectation weight}

Making $\omega$ explicit in (15a) and (15b), one obtains stability intervals with respect to expectation weight, which are affected by the relative slopes $a_{i}$ and by the phase-weight $\nu$. To this end, let us introduce

$$
\begin{aligned}
& \omega_{F, 1}=\frac{2-a_{1}-a_{2}}{\left(1-a_{1}\right)\left(1-a_{2}\right)} \frac{\nu}{2 \nu^{2}-2 \nu+1}-\frac{\sqrt{\Delta_{\omega}}}{\left(1-a_{1}\right)\left(1-a_{2}\right)\left(2 \nu^{2}-2 \nu+1\right)}, \\
& \omega_{F, 2}=\frac{2-a_{1}-a_{2}}{\left(1-a_{1}\right)\left(1-a_{2}\right)} \frac{\nu}{2 \nu^{2}-2 \nu+1}+\frac{\sqrt{\Delta_{\omega}}}{\left(1-a_{1}\right)\left(1-a_{2}\right)\left(2 \nu^{2}-2 \nu+1\right)},
\end{aligned}
$$

provided that

$$
\Delta_{\omega}=\nu^{2}\left(a_{1}+a_{2}-2\right)^{2}-4\left(2 \nu^{2}-2 \nu+1\right)\left(1-a_{1}\right)\left(1-a_{2}\right)
$$

is non-negative, and

$$
\omega_{N S}=\frac{2-a_{1}-a_{2}}{\left(1-a_{1}\right)\left(1-a_{2}\right)} \frac{1}{\nu} .
$$

I notice that $\omega_{F, i}$ are the roots of the l.h.s. of $(15 \mathrm{c})$ and $\omega_{N S}$ is the root of the 1.h.s. of (15b). Depending on the reciprocal positions of $\omega_{F, 1}, \omega_{F, 2}$ and $\omega_{N S}$ we can have different stability scenarios.

The study of steady state stability in the uncoupled model is trivial, as it is a straightforward consequence of the stability condition (13) for the classical cobweb model. In Proposition 3 I briefly summarize the possible stability scenarios for $\nu=1$, both when the relative slopes at the steady state are identical $\left(a_{1}=a_{2}\right)$ and different $\left(\left|a_{1}\right|>\left|a_{2}\right|\right)$. The proof is omitted, since it can be easily inferred from (13).

Proposition 3. Let $\nu=1$. Then

(I) if $\max _{i=1,2}\left|a_{i}\right|<1$, both $p^{i, *}$ are stable for any $\omega \in(0,1]$;

(II) if $\left|a_{1}\right|=\left|a_{2}\right|>1$, both $p^{i, *}$ are stable for $\omega<\bar{\omega}_{1}=\bar{\omega}_{2}$ and unstable for $\omega>\bar{\omega}_{1}=\bar{\omega}_{2}$.

(III) if $\left|a_{1}\right|>1>\left|a_{2}\right|$, both $p^{i, *}$ are stable for $\omega<\bar{\omega}_{1}$, while for $\omega>\bar{\omega}_{1}$ we have that $p^{2, *}$ is unstable and $p^{1, *}$ is stable;

(IV) if $\left|a_{1}\right|>\left|a_{2}\right|>1$, both $p^{i, *}$ are stable for $\omega<\bar{\omega}_{1}$, for $\bar{\omega}_{1}<\omega<\bar{\omega}_{2}$ we have that $p^{2, *}$ is unstable and $p^{1, *}$ is stable, while for $\omega>\bar{\omega}_{2}$ both $p^{i, *}$ are unstable.

In all the previous cases, each $\pi^{i *}$ loses stability through a flip bifurcation.

In the uncoupled model, each component of the steady state equilibrium loses stability for the same value of $\omega$ only if $\left|a_{1}\right|=\left|a_{2}\right|>1$. Just in this particular situation or when the relative slopes $\left|a_{i}\right|$ are both suitably small (in which case both $p^{i, *}$ are stable for any $\omega \in(0,1]$, whether $a_{1}=a_{2}$ or not), we have that for any given expectation weight, both phases locally exhibit the 
same qualitative dynamic behavior, namely they are either both stable or both unstable. Conversely, if relative slopes are different at each $p^{i, *}$ and $\left|a_{1}\right|>1$, we have that for some values of $\omega$ only one $\pi^{i, *}$ is stable.

The main, clear effect of introducing a cyclicity in the demand and supply functions is then to induce, in general, possible asynchrony between the dynamical behavior of the phases, in the sense that, for the same value of the expectation weight, we can have that $\pi^{i, *}$ is stable but $\pi^{j, *}, i \neq j$ is unstable. Now I study what happens when $\nu<1$. I underline that, due to the strongly coupled nature of the dynamical system (8), the above asynchrony is immediately destroyed by a whatever weak coupling $(\nu \lesssim 1)$. The dynamics of both components of $\vec{\pi}$ become synchronous, in the sense that $\pi^{i, *}$ must be either both stable or both unstable. This means that an unconditionally stable phase in the uncoupled model may inherit instability from the other phase. I can say that, even if the coupling does not give rise to a new instability, which is already present in the uncoupled system because of the unstable phase, it allows for spreading it among phases so that it actually has an initial destabilizing effect.

The stability with respect to $\omega$ can be summarized in the following proposition.

Proposition 4. The only possible stability scenarios with respect to $\omega$ are the unconditionally stable, flip destabilizing, Neimark-Sacker destabilizing, mixed and mixed-destabilizing scenarios.

The first significant finding in Proposition 4 is that stability can be potentially lost trough both a flip and a Neimark-Sacker bifurcation. Moreover, in all situations, steady state is stable provided that $\omega$ is suitably small. When the steady state loses stability through a period-doubling bifurcation, a return to stability can occur (mixed scenario) while when stability is lost through a Neimark-Sacker bifurcation, the dynamics stay unstable for any larger expectation weight. Proposition 4 shows that the dynamic behavior of the coupled phases can significantly differ from that of the classical cobweb model. Firstly, instability can occur through a completely different kind of bifurcation, the Neimark-Sacker one. Moreover, when all thresholds $\omega_{F, 1}, \omega_{F, 2}$ and $\omega_{N S}$ belong to $(0,1)$ we have a mixed-destabilizing scenario, which again does not occur in the classical cobweb model. In this situation both stability and instability regions are unconnected and after the return to stability, we can have another subsequent stability loss through a different kind of bifurcation.

I try to summarize the consequences of Proposition 4. The main aspect I want to stress is that the introduction of cyclicity of demand and supply functions, even in the simplest framework of a double phase market, produces a very high level of complexity and ambiguity that cannot be found in the classical cobweb framework.

The first element of complexity is represented by the different dynamical behaviors arising when the steady state loses its stability. As previously recalled, in the classical single phase cobweb model (as well as in the uncoupled double phase one), destabilization can only occur through a flip bifurcation. Conversely, in a double phase market, prices can also exhibit quasi periodic-dynamics when 
a Neimark-Sacker bifurcation arises. For the occurrence of such behavior, the role of demand/supply functions cyclicity and of the particular double phase expectation mechanism can not be completely disentangled. In fact, preliminary numerical investigations show that a sufficiently strong coupling together with a suitably large relative elasticity at both $\pi^{i, *}$ are necessary. The resulting quasi-periodic dynamics are very different from those of a period-doubling destabilization, and provide more intricate and realistic price dynamics as soon as stability is lost. Moreover, in classical cobweb with adaptive expectations, increasing $\omega$ has an unambiguous destabilizing effect and destabilization occurs through a flip bifurcation.

The next element of complexity induced by a double phase market is represented by the presence of multiple stability/instability thresholds, as in the mixed scenarios. This means that stability and instability regions become unconnected, and this has severe consequences on the role of the expectation weight. The most significant effect is that increasing $\omega$ can allow for recovering stability. This is a completely different behavior from the unambiguous destabilizing role of $\omega$ in the classical cobweb model. As shown for example in [6], increasing $\omega$ can only provide a qualitative simplification of the dynamics, which however remain unstable. Conversely, in a double phase cobweb model we have situations in which increasing $\omega$ makes the steady state passing from complex, chaotic dynamics to stable convergent ones, which, as in the mixed scenario, can then remain stable for any larger values of expectation weight, up to $\omega=1$. Moreover, in the mixed-destabilizing scenario, instability can emerge through both a period-doubling and a Neimark-Sacker bifurcations for different values of $\omega$. This means that, for the very same double phase market configuration (namely, for given relative slopes $a_{i}$ ) and with the same degree of coupling among the phases (namely, for the same phase-weight $\nu$ ), different expectation weights can give rise to either periodic and even chaotic or quasi-periodic dynamics.

The only aspect shared with classical cobweb is that there always exists a sufficiently small positive $\tilde{\omega}$ so that the steady state is stable for any $\omega \in(0, \tilde{\omega})$.

\subsection{Stability with respect to phase weight}

Stability with respect to phase weight can be studied by making $\nu$ explicit in (15). Also in this case, we find three stability thresholds, obtained by solving $(15 \mathrm{c})$

$$
\begin{aligned}
\nu_{F, 1} & =\frac{1}{2}-\frac{\left(a_{1}+a_{2}-2\right)}{2\left(a_{1}-1\right)\left(a_{2}-1\right) \omega}-\frac{\sqrt{\Delta_{\nu}}}{2\left(a_{1}-1\right)\left(a_{2}-1\right) \omega} \\
\nu_{F, 2} & =\frac{1}{2}-\frac{\left(a_{1}+a_{2}-2\right)}{2\left(a_{1}-1\right)\left(a_{2}-1\right) \omega}+\frac{\sqrt{\Delta_{\nu}}}{2\left(a_{1}-1\right)\left(a_{2}-1\right) \omega},
\end{aligned}
$$

provided that

$$
\begin{aligned}
\Delta_{\nu}= & -\left(a_{1}-1\right)\left(a_{2}-1\right)^{2} \omega^{2}-2\left(a_{1}-1\right)\left(a_{2}-1\right)\left(a_{1}+a_{2}-2\right) \omega \\
& -8\left(a_{1}-1\right)\left(a_{2}-1\right)+\left(a_{1}+a_{2}-2\right)^{2}
\end{aligned}
$$


is non-negative, and by solving (15b)

$$
\nu_{N S}=-\frac{\left(a_{1}+a_{2}-2\right)}{\left(a_{1}-1\right)\left(a_{2}-1\right)} \frac{1}{\omega} .
$$

I notice that $\nu_{F, 1}<\nu_{F, 2}$ are the roots of the l.h.s. of (15c) and $\nu_{N S}$ is the root of the l.h.s. of (15b). The possible stability scenarios are briefly summarized in the following proposition.

Proposition 5. The only possible stability scenarios with respect to $\nu$ are the unconditionally stable, flip destabilizing, Neimark-Sacker destabilizing and unconditionally unstable scenarios.

Proposition 5 shows that the role of $\nu$ is in general destabilizing. Except for the unconditionally stable and unstable scenarios, increasing $\nu$ has the sole effect of introducing instability in the dynamics, which can potentially occur by either flip or Neimark-Sacker bifurcations. On the other hand, mixed scenarios are not possible with respect to the phase-weight.

Even if the role of $\nu$ is then much less ambiguous than that of $\omega$, I notice that it can be affected by the other parameters in an ambiguous way. Firstly, the double possible route toward instability is straightforwardly determined by the joint effect of $\omega$ and $a_{i}$. Finally, I stress that not for any parameter configurations is possible to stabilize dynamics by letting $\nu \rightarrow 1 / 2$, namely by considering an (approximatively) uniform average of the in-phase and out-of-phase forecasting errors, as the occurrence of unconditionally unstable scenarios shows.

\subsection{Stability with respect to relative slopes}

Concerning $a_{i}$, as in the classical cobweb theorem, in a double phase model steady state stability depends on the relative slopes of supply and demand functions. In the present case, stability is indeed affected in a more complicated way by two, generally different, relative slopes. To this end, I notice that we can rephrase (13) into

$$
-1<\frac{\omega}{2}\left(\frac{S^{\prime}\left(p^{*}\right)}{D^{\prime}\left(p^{*}\right)}-1\right),
$$

in which the right hand side corresponds to the slope at the steady state of the evolution function obtained in the classical cobweb model with adaptive expectations. I then rewrite stability conditions (15a) and (15b) by introducing constants $k_{1}$ and $k_{2}$, defined by

$$
k_{i}=\frac{\omega}{2}\left(a_{i}-1\right), i \in\{1,2\} .
$$

We obtain system

$$
\left\{\begin{array}{l}
\left(k_{1}+k_{2}\right)+2 \nu k_{1} k_{2}<0 \\
k_{1} k_{2}\left(2 \nu^{2}-2 \nu+1\right)+\nu\left(k_{1}+k_{2}\right)+1>0
\end{array}\right.
$$



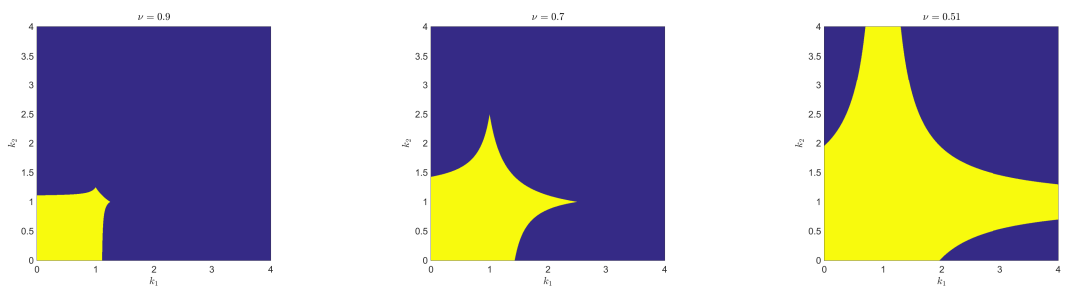

Figure 2: Stability regions (in yellow) with respect to $k_{i}$ for different values of $\nu$. The stability region becomes larger as $\nu$ decreases.

which can not be explicitly solved with respect to $k_{i}$ but which can be easily investigated graphically. I report in Figure 2 the plots of three stability regions in $\left(k_{1}, k_{2}\right)$-plane for different values of $\nu$. As we can see, the stability region becomes larger as $\nu$ decreases, in agreement with the previous results about $\nu$. More surprisingly, I notice that we can have situations in which decreasing the value of $k_{1}$ while keeping fixed $k_{2}$ (and vice versa) dampens stability. As an example, if we take $k_{2}=2$, we have that $\vec{p}^{*}$ is stable for $k_{1} \approx 1$ but not for smaller values.

\section{Conclusions}

In this paper, a cobweb model for a double phase market was introduced and studied. This required a new approach, especially for the expectation formation mechanism, as the classical cobweb model is not sufficient to describe such class of markets. The result was a non-autonomous difference equation with a steady cycle of period 2. Stability of the corresponding vector steady state for an associated two-dimensional autonomous system is analyzed, showing that it can potentially exhibit completely different dynamics from the classical cobweb model under adaptive expectations. Firstly, expectation weight can have a much more ambiguous role, as decreasing it can make stable dynamics become unstable. Instability can now give rise to more realistic quasi-periodic dynamics, through a Neimark-Sacker bifurcation. Moreover, the presence of up to three stability thresholds allows for the occurrence of both kinds of instability for different values of $\omega$, while keeping unchanged the economic context. The role of phase weight conversely seems less ambiguous, as $\nu$ has a general destabilizing role. This means that coupling the dynamics of each market phase has a stabilizing effect. Finally, the role of relative slopes at the equilibrium is ambiguous, too, as it is no more true that less steep relative slopes mean more stable dynamics. The proposed approach can be applied to general situations, in which the considered market is characterized by $M>2$ phases. Moreover, in this paper stability investigation, studied with respect to each parameter $\omega, \nu$ and $a_{i}$ on its own, aimed to show the possible occurrence of new scenarios. Preliminary numerical simulations show that each predicted scenario can actually occur. It 
would be desirable to deepen the analysis, to understand the reciprocal effects of the considered parameters, as well as the specific conditions under which each scenario actually occurs. Finally, it would be interesting to investigate the capabilities of the proposed approach to describe peculiar dynamical aspects of a real world situation.

\section{Appendix}

Proof of Proposition 1. Firstly, I prove that $\vec{p}^{*}=\Phi_{2}\left(\Phi_{1}\left(\vec{p}^{*}\right)\right)$. Recalling that $\vec{p}^{*}$ satisfies $(6)$, we have $\Phi_{2}\left(\Phi_{1}\left(\vec{p}^{*}\right)\right)=\Phi_{2}\left(p^{2, *}, p^{1, *}\right)=\left(p^{1, *}, p^{2, *}\right)$, which allows concluding the first part of the proof. Now we need to show that if $p=\Phi_{2}\left(\Phi_{1}(p)\right)$, then $p=\vec{p}^{*}$. To this end, let us suppose that

$$
\Phi_{1}\left(p^{1}, p^{2}\right):\left\{\begin{array}{l}
\rho^{1}=p^{2}+\omega(1-\nu) \delta_{1} \\
\rho^{2}=p^{1}+\omega \nu \delta_{1}
\end{array}\right.
$$

and

$$
\Phi_{2}\left(\rho^{1}, \rho^{2}\right):\left\{\begin{array}{l}
p^{1}=\rho^{2}+\omega(1-\nu) \delta_{2} \\
p^{2}=\rho^{1}+\omega \nu \delta_{2}
\end{array}\right.
$$

where I set

$$
\begin{aligned}
& \delta_{1}=D_{1}^{-1}\left(S\left(p^{1}\right)\right)-p^{1}, \\
& \delta_{2}=D_{2}^{-1}\left(S\left(\rho^{1}\right)\right)-\rho^{1} .
\end{aligned}
$$

Combining (18) and (19) we obtain

$$
\begin{aligned}
& (1-\nu) \delta_{1}+\nu \delta_{2}=0, \\
& \nu \delta_{1}+(1-\nu) \delta_{2}=0,
\end{aligned}
$$

which is an homogeneous square linear system, whose coefficient matrix $A=$ $(1-\nu, \nu ; \nu, 1-\nu)$ has $\operatorname{det}(A) \neq 0$, since $\nu \neq 1 / 2$. This means that its unique solution is $\left(\delta_{1}, \delta_{2}\right)=(0,0)$. Recalling $(6)$, from $\delta_{1}=0$ we have that $p^{1}=p^{1, *}$, while from $\delta_{2}=0$ we have that $\rho^{1}=p^{2, *}$. Inserting $\rho^{1}=p^{2, *}$ into the first equation in (18) and using $\delta_{1}=0$ we obtain $p^{2}=p^{2, *}$, which concludes the proof.

Proof of Proposition 2. Let $J_{T}(\vec{\pi})$ be the Jacobian matrix of map $T$ defined by (8) and let $J_{T}^{*}=J_{T}\left(\vec{p}^{*}\right)$. Recalling that $\vec{p}^{*}$ is locally asymptotically stable provided that the eigenvalues $\lambda_{i}$ of $J_{T}^{*}$ satisfy $\left|\lambda_{i}\right|<1$, I start noticing that

$$
J_{T}(\vec{\pi})=J_{\Phi_{2}}\left(\Phi_{1}(\vec{\pi})\right) J_{\Phi_{1}}(\vec{\pi})
$$

where $J_{\Phi_{i}}(\vec{\pi})$ is the Jacobian matrix of function $\Phi_{i}$. If $\vec{\pi}=\left(\pi^{1}, \pi^{2}\right)$, we have

$$
J_{\Phi_{i}}(\vec{\pi})=\left(\begin{array}{cc}
\omega \nu_{2}\left(\left(D_{i}^{-1}\right)^{\prime}\left(S\left(\pi^{1}\right)\right) S^{\prime}\left(\pi^{1}\right)-1\right) & 1 \\
1+\omega \nu_{1}\left(\left(D_{i}^{-1}\right)^{\prime}\left(S\left(\pi^{1}\right)\right) S^{\prime}\left(\pi^{1}\right)-1\right) & 0
\end{array}\right)
$$


Since $\Phi_{1}\left(\pi^{1, *}, \pi^{2, *}\right)=\left(\pi^{2, *}, \pi^{1, *}\right)$, noticing that $\left(D_{i}^{-1}\right)^{\prime}\left(S\left(\pi^{i, *}\right)\right)=1 / D^{\prime}\left(\pi^{i, *}\right)$, we have

$$
\begin{aligned}
J_{\Phi_{1}}\left(\pi^{1, *}, \pi^{2, *}\right) & =\left(\begin{array}{cc}
\omega \nu_{2}\left(a_{1}-1\right) & 1 \\
1+\omega \nu_{1}\left(a_{1}-1\right) & 0
\end{array}\right), \\
J_{\Phi_{2}}\left(\Phi_{1}\left(\pi^{1, *}, \pi^{2, *}\right)\right) & =\left(\begin{array}{cc}
\omega \nu_{2}\left(a_{2}-1\right) & 1 \\
1+\omega \nu_{1}\left(a_{2}-1\right) & 0
\end{array}\right)
\end{aligned}
$$

from which we obtain

$$
J^{*}=\left(\begin{array}{cc}
\nu \omega\left(a_{1}-1\right)+\omega^{2}\left(a_{1}-1\right)\left(a_{2}-1\right)(\nu-1)^{2}+1 & -\omega\left(a_{2}-1\right)(\nu-1) \\
-\omega\left(a_{1}-1\right)(\nu-1)\left(a_{2} \nu \omega-\nu \omega+1\right) & \nu \omega\left(a_{2}-1\right)+1
\end{array}\right)
$$

where I used (14). Since $\left|\lambda_{i}\right|<1$ provided that

$$
\left\{\begin{array}{l}
1-\operatorname{Tr}\left(J^{*}\right)+\operatorname{det}\left(J^{*}\right)>0 \\
1-\operatorname{det}\left(J^{*}\right)>0 \\
1+\operatorname{Tr}\left(J^{*}\right)+\operatorname{det}\left(J^{*}\right)>0
\end{array}\right.
$$

from

$$
\operatorname{Tr}\left(J^{*}\right)=\nu \omega\left(a_{1}+a_{2}-2\right)+\omega^{2}\left(a_{1}-1\right)\left(a_{2}-1\right)(\nu-1)^{2}+2
$$

and

$$
\operatorname{det}\left(J^{*}\right)=\left(\nu \omega\left(a_{1}-1\right)+1\right)\left(\nu \omega\left(a_{2}-1\right)+1\right)
$$

System (20) becomes

$$
\left\{\begin{array}{l}
\omega^{2}(2 \nu-1)\left(1-a_{1}\right)\left(1-a_{2}\right)>0 \\
1-\left(\left(a_{1}-1\right) \nu \omega+1\right)\left(\left(a_{2}-1\right) \nu \omega+1\right)>0 \\
\left(\left(a_{1}-1\right) \nu \omega+1\right)\left(\left(a_{2}-1\right) \nu \omega+1\right) \\
+\nu \omega\left(a_{1}-1\right)+\nu \omega\left(a_{2}-1\right)+\omega^{2}\left(a_{1}-1\right)\left(a_{2}-1\right)(\nu-1)^{2}+3>0
\end{array}\right.
$$

which easily provides (15).

Lemma 1. Let $\nu>1 / 2$. Then

- $2 \nu^{2}-2 \nu+1>0$

- $\omega_{N S}>0$

- if $\Delta_{\omega}>0$, then $0<\omega_{F, 1}<\omega_{F, 2}$ and $\omega_{F, 1}<w_{N S}$.

Proof. The first two statements are trivial. If $\Delta_{\omega}>0$, since the coefficient of $\omega^{2}$ in (15c) is positive, we have $\omega_{F, 1}<\omega_{F, 2}$. Moreover, we can rewrite $\omega_{F, 1}>0$ into the equivalent form

$$
-\left(a_{1}+a_{2}-2\right) \nu>\sqrt{\Delta_{\omega}},
$$

which, if $\Delta_{\omega}>0$ and since both sides are positive, becomes

$$
4\left(a_{1}-1\right)\left(a_{2}-1\right)\left(2 \nu^{2}-2 \nu+1\right)>0,
$$


which is fulfilled. To prove the last inequality it is sufficient to notice that $\omega_{F, 1}<w_{N S}$ can be equivalently rewritten as

$$
\frac{\left(a_{1}+a_{2}-2\right)}{\left(a_{1}-1\right)\left(a_{2}-1\right)}\left(\frac{(1-\nu)^{2}}{2 \nu^{2}-2 \nu+1}\right)<\frac{\nu \sqrt{\Delta_{\omega}}}{\left(a_{1}-1\right)\left(a_{2}-1\right)\left(2 \nu^{2}-2 \nu+1\right)},
$$

which is indeed true since its l.h.s. is non positive and its r.h.s. is strictly positive.

Proof of Proposition 4. Firstly, I notice that, since the coefficient of $\omega^{2}$ is positive (see Lemma 1), the second condition of (3) is solved by $w \in\left(0, \omega_{F, 1}\right) \cup$ $\left(\omega_{F, 2}, 1\right]$ when $\Delta_{\omega} \geq 0$, while it is always true when $\Delta_{\omega}<0$.

The unconditionally stable scenario is realized if $\Delta_{\omega}<0$ and $\omega_{N S}>1$ or if $\Delta_{\omega} \geq 0$ and $\omega_{F, 1}>1$, as in this case Lemma 1 guarantees that both $\omega_{F, 2}$ and $\omega_{N S}$ belong to $(1,+\infty)$.

The Neimark-Sacker destabilizing scenario arises when $\Delta_{\omega}<0$ and $\omega_{N S}<1$. I notice that no other scenarios are possible when $\Delta_{\omega}<0$.

The remaining scenarios require $\Delta>0$. If $\omega_{F_{1}}<1<\omega_{F, 2}$, since from Lemma 1 we have $\omega_{F_{1}}<\omega_{N S}$, or if $\omega_{N S}<\omega_{F, 2}<1$, we obtain the flip destabilizing scenario. Finally, if $\omega_{F, 2}<1<\omega_{N S}$ we have the mixed scenario, while if $\omega_{F, 2}<\omega_{N S}<1$ we have the mixed destabilizing scenario.

If we set $a_{1}=a_{2}$, we have that (16) reduces to

$$
4 \nu^{2}\left(1-a_{1}\right)^{2}-4\left(2 \nu^{2}-2 \nu+1\right)\left(1-a_{1}\right)^{2}=4\left(1-a_{1}\right)^{2}(1-\nu)^{2},
$$

which is positive for $\nu<1$. Then, condition (15c) is always satisfied and the possible scenarios are determined by condition (15b), which allows concluding.

Lemma 2. Let $\omega \in(0,1]$. Then

- if $\Delta_{\nu>0}$, then $\nu_{F, 1}<\nu_{F, 2}$;

- if $\Delta_{\nu>0}$ and $\nu_{F, 2}<1$, then $\nu_{N} \leq \nu_{F, 2}$.

Proof. The former statement is straightforward. To prove the latter, we notice that if we impose $\nu_{F, 1}<\nu_{N}$ we easily come to $\left(a_{1}-1\right)\left(a_{2}-1\right) \omega+\left(a_{1}+a_{2}-\right.$ $2)+\sqrt{\Delta_{\nu}}<0$, which necessarily requires $\left(a_{1}-1\right)\left(a_{2}-1\right) \omega+\left(a_{1}+a_{2}-2\right)<0$. However, $\nu_{N}<1$ requires $\left(a_{1}-1\right)\left(a_{2}-1\right) \omega+\left(a_{1}+a_{2}-2\right)>0$, which is impossible. This excludes $\nu_{F, 1}<\nu_{N}<1$. Similarly, if we impose $\nu_{F, 2}<1$ we obtain $\sqrt{\Delta_{\nu}}<\left(a_{1}-1\right)\left(a_{2}-1\right) \omega+\left(a_{1}+a_{2}-2\right)$, which necessarily requires $\left(a_{1}-1\right)\left(a_{2}-1\right) \omega+\left(a_{1}+a_{2}-2\right)>0$. However, $\nu_{N}>1$ requires $\left(a_{1}-1\right)\left(a_{2}-\right.$ $1) \omega+\left(a_{1}+a_{2}-2\right)<0$, which is impossible. This concludes the proof.

From the previous lemma we have that, since neither $1 / 2<\nu_{F, 1}<\nu_{F, 2}<$ $\nu_{N}<1$ nor $\nu_{F, 1}<\nu_{F, 2}<1<\nu_{N}$ are possible, no mixed/mixed destabilizing scenarios can occur with respect to $\nu$. 
Proof of Proposition 5. The unconditionally stable scenario occurs when $\nu_{N S} \geq$ 1 and either $\Delta_{\nu}<0$ or $\Delta_{\nu} \geq 0$ and $\nu_{F, 1} \geq 1$. Conversely, the equilibrium is unstable for any $\nu$ when either $\Delta_{\nu}<0$ and $\nu_{N S} \leq 1 / 2$ or $\Delta_{\nu} \geq 0$ and $\nu_{F, 1} \leq 1 / 2$. The flip destabilizing scenario requires $\nu_{F, 1} \in(1 / 2,1)$ and $\nu_{N S}>\nu_{F, 1}$, while the Neimark-Sacker destabilizing scenario arises when $\nu_{N S} \in(1 / 2,1)$ and either $\Delta_{\nu}<0$ or $\Delta_{\nu} \geq 0$ and $\nu_{N S}<\nu_{F, 1}$.

\section{References}

[1] N. Kaldor. A classificatory note on the determination of equilibrium. Review of Economic Studies, 1:122-136, 1934.

[2] M. Ezekiel. The cobweb theorem. Quarterly Journal of Economics, 52:255$280,1938$.

[3] M. Nerlove. Adaptive expectations and cobweb phenomena. Quarterly Journal of Economics, 72:227-240, 1958.

[4] C. Chiarella. The cobweb model. Its instability and the onset of chaos. Economic Modelling, 5:377-384, 1988.

[5] C. H. Hommes. Adaptive learning and roads to chaos. The case of the cobweb. Economics Letters, 36:127-132, 1991.

[6] C. H. Hommes. Dynamics of the cobweb model with adaptive expectations and nonlinear supply and demand. Journal of Economic Behavior 8 Organization, 24(3):315 - 335, 1994.

[7] R. Dieci and F. Westerhoff. Interacting cobweb markets. Journal of Economic Behavior \& Organization, 75:461-481, 2010.

[8] L. Lundberg, E. Jonson, K. Lindgren, D. Bryngelsson, and V. Verendel. A cobweb model of land-use competition between food and bioenergy crops. Journal of Economic Dynamics and Control, 53:1 - 14, 2015.

[9] F. Cavalli, A. Naimzada, and L. Parisio. A cobweb model for electricity markets. In International Conference on the European Energy Market, EEM, volume 2015, 2015.

[10] G. I. Bischi and A. Naimzada. Global analysis of a nonlinear model with learning. Economic Notes, 26:143-174, 1997.

[11] F. Cavalli and A. Naimzada. A tatonnement process with fading memory, stabilization and optimal speed of convergence. Chaos, Solitons and Fractals, 79:116-129, 2015.

[12] G. I. Bischi, F. Cavalli, and A. Naimzada. Mann iteration with power means. Journal of Difference Equations and Applications, 21(12):1212$1233,2015$. 
[13] J. A. Carlson. An invariably stable cobweb model. Review of Economic Studies, 35:360-362, 1968.

[14] J. M. Holmes and R. Manning. Memory and market stability. The case of the cobweb. Economics Letters, 28:1-7, 1988.

[15] J. M. Gaffney and C. E. M. Pearce. Memory, market, stability and the nonlinear cobweb theorem. ANZIAM Journal, 45:547-555, 2004.

[16] J. M. Gaffney and C. E. M. Pearce. Nonlinear-cobweb dynamics in the approach to equilibrium. ANZIAM Journal, 46:79-83, 2004.

[17] C. H. Hommes. Behavioral Rationality and Heterogeneous Expectations in Complex Economic Systems. Cambridge University Press, 2013.

[18] R. Manning. A generalization of a cobweb theorem. Review of Economic Studies, 36:123-125, 1971.

[19] S. Wiggins. Introduction to Applied Nonlinear Dynamical Systems and Chaos. Texts in Applied Mathematics, Springer-Verlag, New York, 1990. 